\title{
Fast algorithms for chiral fermions in 2 dimensions
}

\author{
Dafina Hyka $\left(\right.$ Xhako) ${ }^{1, \star}$ and Rudina Osmanaj (Zeqirllari) ${ }^{2}$ \\ ${ }^{1}$ Polytechnic University of Tirana, Faculty of Mathematical Engineering and Physical Engineering, Depart- \\ ment of Physics \\ ${ }^{2}$ University of Tirana, Faculty of Natural Sciences, Department of Physics
}

\begin{abstract}
In lattice QCD simulations the formulation of the theory in lattice should be chiral in order that symmetry breaking happens dynamically from interactions. In order to guarantee this symmetry on the lattice one uses overlap and domain wall fermions. On the other hand high computational cost of lattice QCD simulations with overlap or domain wall fermions remains a major obstacle of research in the field of elementary particles. We have developed the preconditioned GMRESR algorithm as fast inverting algorithm for chiral fermions in U(1) lattice gauge theory. In this algorithm we used the geometric multigrid idea along the extra dimension.The main result of this work is that the preconditioned GMRESR is capable to accelerate the convergence 2 to 12 times faster than the other optimal algorithms (SHUMR) for different coupling constant and lattice $32 \times 32$. Also, in this paper we tested it for larger lattice size $64 x 64$. From the results of simulations we can see that our algorithm is faster than SHUMR. This is a very promising result that this algorithm can be adapted also in 4 dimension.
\end{abstract}

\section{Introduction}

Lattice QCD is a lattice gauge theory formulated on a lattice of points in space and time. Fields representing quarks are defined at lattice sites and the gluon fields are defined on the links connecting neighbouring sites. To study the interactions between quarks should be calculated mathematically the quark propagator which is the inverse of the Dirac operator. It is important to build in lattice a theory with chiral fermions as chiral symmetry is characteristic of strong interactions. Therefore it should be made inversion of the chiral Dirac operator in the lattice which has high complexity [14]. On the other hand, as the lattice volume is increased and simulations are repeated for a large number of statistically independent configurations, it significantly increases the need for computational power. An efficient way to solve this problem is development of fast inversion algorithms in order to gain time and computational cost. In this work we develop in fact prototypes of algorithms that are using high-end computing as our contribution to QCDLAB package [2],[3] for simulating chiral fermions. This package, a MATLAB/OCTAVE based environment, allows fast prototyping of linear algebraic computations and thus accelerates the process of finding the most efficient fermion algorithm.

${ }^{\star}$ Speaker, e-mail: dafinaxhako@yahoo.com 


\section{Chiral fermions on the lattice}

The simulation of LQCD with chiral fermions is very important for the development of the theory of elementary particles. There are two formulation of chiral fermions on the lattice:

- Domain wall fermions [10], [9]

- Overlap fermions [14],[12],[11]

This two formulations are closely related [1], the truncated overlap variant of domain wall fermions [4] are equivalent to overlap fermions at any lattice spacing in four dimensions [5]. The main challenge in numerical simulations of LQCD with chiral fermions is solving large linear system of equations:

$$
D x=b
$$

where $D$ is the chiral Dirac operator (a sparse and large matrix), $b$ the right side or the source of quarks and $x$ are the quark propagator. The chiral Dirac operator is representing by the Neuberger operator (overlap operator), which is a shifted unitary matrix of the form [13].

$$
D_{N}=c_{1} I-c_{2} V
$$

where $V=A\left(A^{+} A\right)^{-\frac{1}{2}}$ is a unitary matrix, $I$ unit matrix and $A=M-a D_{W} . D_{W}$ is Wilson-Dirac operator which has form:

$$
D_{W}=\frac{1}{2} \Sigma_{\mu}\left[\gamma_{\mu}\left(\partial_{\mu}{ }^{*}+\partial_{\mu}\right)-a \partial_{\mu}^{*} \partial_{\mu}\right]
$$

where $\partial_{\mu}$ and $\partial_{\mu}{ }^{*}$ are the nearest-neighbour forward and backward difference operators. These operators are $3 \times 3$ unitary matrices with determinant one. A set of these matrices forms a configuration. $\gamma_{\mu}$, $(\mu=1, \ldots, 5)$ are $4 x 4$ matrices related to the spin of particles.

Also, the Neuberger operator can be written from the sign function [13] as below:

$$
D_{N}=c_{1} I-c_{2} \gamma_{5} \operatorname{sign}\left(H_{W}\right)
$$

where $H_{W}=\gamma_{5}\left(M-a D_{W}\right)$.

In order that sign function to be non-trivial, the Wilson-Dirac operator should be indefinite. Thus, its bare mass $M$ should be sufficiently negative, usually it is taken in the interval $(-2,0) . a$ is the lattice spacing, $c_{1}$ and $c_{2}$ are two constants that are related with bare quark mass $m_{q}$ from:

$$
c_{1}=\frac{1+m_{q}}{2}, c_{2}=\frac{1-m_{q}}{2}
$$

\section{Development of algorithms in 2 dimensions}

Simulation of lattice theory with U(1) group symmetry, the case of the Quantum Electrodynamics, in two dimensional space-times has always been a testing ground for development of new algorithms. The complexity of chiral Dirac operator is now of order $2 N$, where $N$ are the lattice points. For development of new algorithms we use QCDLAB, which is a research tool for lattice QCD algorithms [2], [3]. We use here, the version QCDLAB 1.0 [2] with the Schwinger model on the lattice, which shares many features and algorithms with lattice QCD. The time of inversion in the standard Krylov's inversion algorithms [7] escalate with the inverse of quark mass known as critical slowdown. Thus arises the need of building an inversion algorithm for chiral fermions that avoids critical slowing. One method to reduce the critical slowing down is to use the preconditioned algorithms [7].In [8] the preconditioned is built upon an inaccurate approximation to the sign function, while we use as the 
preconditioned part the approximation of the overlap operator with the truncated overlap operator with finite $N_{3}$ dimension. Thus, in order to develop fast algorithms, we use the truncated overlap variant of domain wall fermions in $2+1$ dimensions with the extra finite dimension $N_{3}$. Using this idea we have developed an algorithm called the preconditioned GMRESR in [15] which converges faster than optimal algorithms used in simulations with chiral fermions.In essence the preconditioned GMRESR follows two levels;

- First level: The preconditioned part. Compute approximate solution for the linear system using the equivalent of the overlap operator, the truncated overlap operator in $2+1$ dimensions with an extra finite $N_{3}$ dimension using small accuracy. In this case we have used the CGNE (Conjugate Gradients on Normal Equations) algorithm as an known optimal solver.

- Second level:Find the true residual using the real overlap operator in $N_{3}$ infinite dimension, and use this residual to control the residual of the first step in each iteration. These two levels will continue in cycle still is reached a specific tolerance.

In [15] this algorithm is developed for a single coupling constant of the gauge field in $32 \times 32$ lattice volume. In this paper we test it for larger lattice volume $64 x 64$. For compare purposes, we use also another optimal inversion algorithm of chiral fermions such as SHUMR (Shifted Unitary Minimal Residual) [6]. We have carried out numerical simulations for 100 statistically independent gauge field configurations with three values of coupling constant $\beta=1.0, \beta=1.1$ and $\beta=1.2$ in $64 x 64$ lattice volume using the preconditioned GMRESR.

\section{Results}

For each coupling constant simulations are performed for different quark masses $m_{q}=$ $[0.5,0.45,0.4,0.35,0.3,0.25,0.2,0.15,0.1,0.05,0.01]$. The same numerical simulations are performed with SHUMR algorithm. We have compared the convergence history of the residual norm of the preconditioned GMRESR algorithm with the SHUMR algorithm. We have calculated the number of the Wilson-Dirac operator multiplications till convergence and the residual norm for 100 independent gauge fields configurations. In Figure 1, Figure 2, Figure 3 we present graphical results of the history of the convergence for each algorithm for three values of coupling constant and for quark mass 0.01 . 


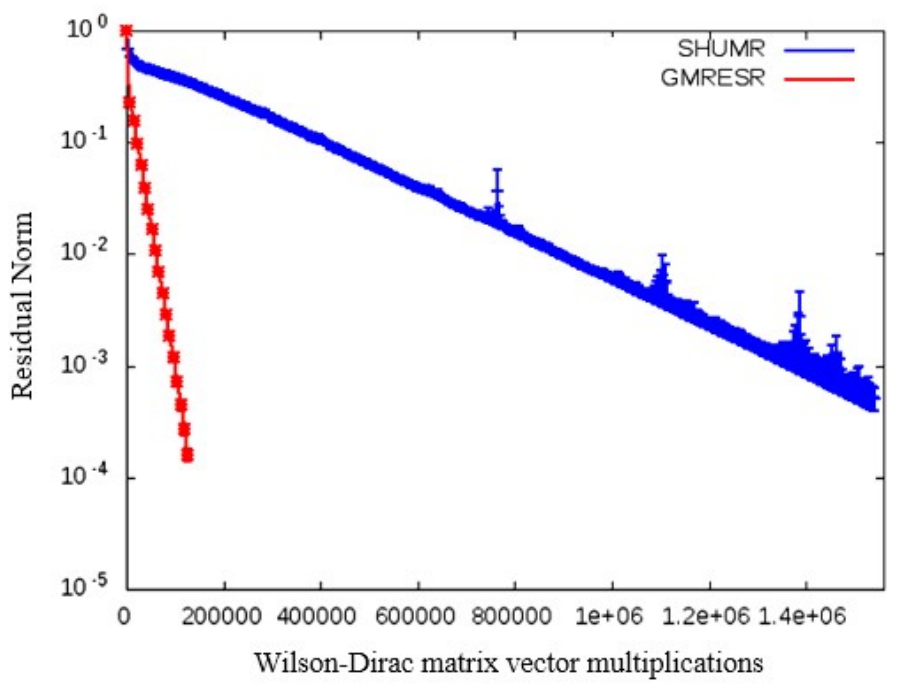

Figure 1. The convergence history of the residual norm as the function of the number of Dirac-Wilson multiplications for SHUMR and the preconditioned GMRESR inverting chiral Dirac operator on $64 x 64$ lattice background $\mathrm{U}(1)$ field at coupling constant $\beta=1.0$ and quark mass $m_{q}=0.01$ (in lattice unit).

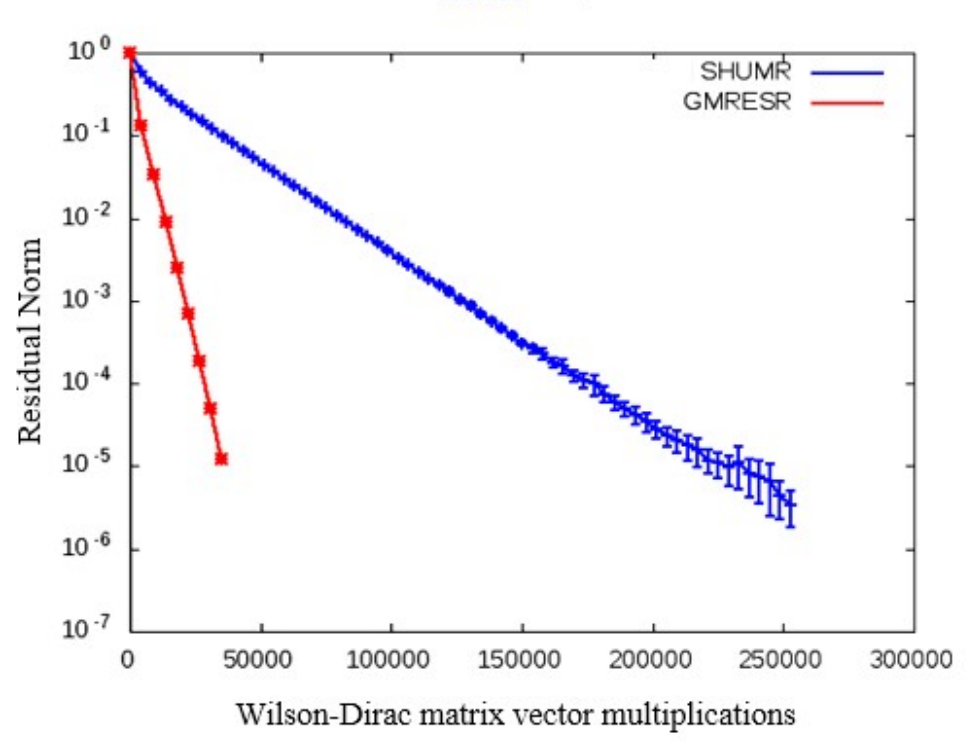

Figure 2. The convergence history of the residual norm as the function of the number of Dirac-Wilson multiplications for SHUMR and the preconditioned GMRESR inverting chiral Dirac operator on $64 x 64$ lattice background $\mathrm{U}(1)$ field at coupling constant $\beta=1.1$ and quark mass $m_{q}=0.01$ (in lattice unit). 


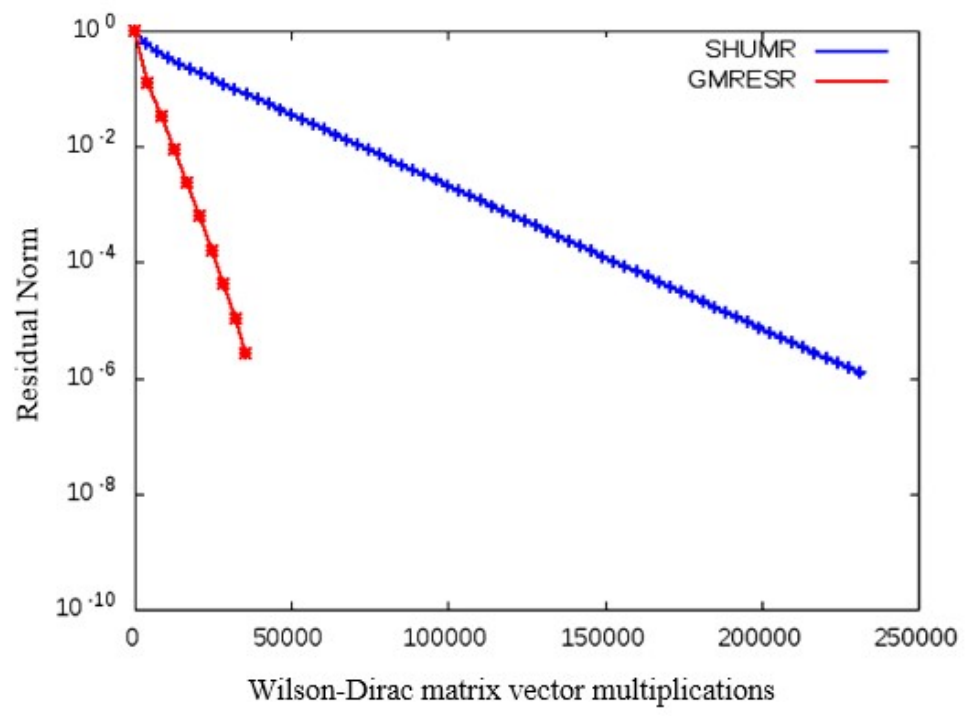

Figure 3. The convergence history of the residual norm as the function of the number of Dirac-Wilson multiplications for SHUMR and the preconditioned GMRESR inverting chiral Dirac operator on $64 x 64$ lattice background $\mathrm{U}(1)$ field at coupling constant $\beta=1.2$ and quark mass $m_{q}=0.01$ (in lattice unit).

As we can see from graphical results in Figures 1,2, 3, the preconditioned GMRESR algorithm converges faster than SHUMR for different coupling constant. From Figure 1 for quark mass 0.01 and coupling constant 1.0 we can save a factor of 12. From Figure 2 for quark mass 0.01 and coupling constant 1.1 we can save a factor of 7. From Figure 3 for quark mass 0.01 and coupling constant 1.2 we can save a factor of 7. Also for different coupling regimes the preconditioned GMRESR algorithm converges faster than SHUMR almost with the same factor.

\section{Conclusions}

The preconditioned GMRESR algorithm is the most promising inversion algorithm for LQCD simulations with chiral fermions. We first proposed this algorithm in [15]. In this paper we tested it for different coupling constant on $64 x 64$ lattice volume. It showed that the preconditioned GMRESR converges quickly, 2 to 12 faster than the optimal algorithm SHUMR even for different coupling constant, which makes the results of this algorithm more stable. This are very promising results to developed in the future the preconditioned GMRESR algorithm in 4 dimensional QCD, so in SU(3).

\section{Acknowledgement}

Acknowledgements goes to the serves in the parallel system of the Faculty of Information Technology, Polytechnic University of Tirana. 


\section{References}

[1] A. Boriçi. "Computational methods for the fermion determinant and the link between overlap and domain wall fermions". In: QCD and numerical analysis III. Proceedings, 3rd International Workshop, Edinburgh, UK, June 30-July 4, 2003 (2004), pp. 25-39. arXiv: heplat/0402035 [hep-lat].

[2] A. Boriçi. “QCDLAB: Designing Lattice QCD Algorithms with MATLAB”. In: Journal of High Energy Physics 2006 (2006), p. 24. eprint: hep-lat/0610054.

[3] A. Boriçi. "Speeding up domain wall fermion algorithms using QCDLAB”. In: Journal of High Energy Physics 2007 (2007). Invited talk at Conference: Workshop on Domain Wall Fermions at Ten Years, p. 06. eprint: hep-lat/0703021.

[4] A. Boriçi. “Truncated overlap fermions”. In: Nucl.Phys.Proc.Suppl. 83 (2000), pp. 771-773. DOI: 10.1016/S0920-5632(00)91802-4.

[5] A. Boriçi. "Truncated Overlap Fermions: the link between Overlap and Domain Wall Fermions”. In: NATO ASI series C, Mathematical and physical sciences; 553 (1999). Proocedings, NATO Advanced Research Workshop on Lattice Fermions and Structure of the Vacuum,Dubna, Russia, October 5-9, pp. 42-52. eprint: hep-lat/9912040.

[6] A. Boriçi and A. Allkoci. "A fast minimal residual solver for overlap fermions". In: Journal of High Energy Physics 2006 (2007), p. 27. eprint: hep-lat/0602015.

[7] A. Boriçi and P. De Forcrand. "Fast Krylov space methods for calculation of quark propagator". In: Physics computing '94. (1994). Proceedings, 6th Joint EPS-APS International Conference, PC'94, Lugano, Switzerland, August 22-26, 1994, pp. 711-714. arXiv: hep-lat/9405001.

[8] N. Cundy et. al. "Numerical Methods for the QCD Overlap Operator:III. Nested Iterations". In: Comput.Phys.Commun. 165.1 (1974), p. 22.

[9] V. Furman and Y. Shamir. "Axial symmetries in lattice QCD with Kaplan fermions". In: Nuclear Physics B439.1 (1995), pp. 54-78. DoI: 10.1016/0550-3213(95)00031-M.

[10] D. Kaplan. "A Method for Simulating Chiral Fermions on the Lattice”. In: Phys.Lett. B228.1 (1992), p. 342. Dor: 10.1016/0370-2693(92)91112-M.

[11] R. Narayanan and H. Neuberger. "A construction of lattice chiral gauge theories". In: Nucl.Phys. B 443.1 (1995), p. 305. Dor: 10.1016/0550-3213(95)00111-5.

[12] R. Narayanan and H. Neuberger. "Infinitely many regulator fields for chiral fermions". In: Phys.Lett. B302 (1993), pp. 62-69. Dor: 10.1016/0370-2693(93)90636-V.

[13] H. Neuberger. "Exactly massless quarks on the lattice". In: Phys. Lett. B 417.1 (1998), p. 141. DoI: 10.1016/S0370-2693(97)01368-3.

[14] H. Neuberger. "Overlap lattice Dirac operator and dynamical fermions". In: Physical Review D 60.6 (1999), p. 065006.

[15] D. Xhako and A. Boriçi. "Fast Algorithms for Simulating Chiral Fermions in U(1) Lattice Gauge Theory". In: American Journal of Physics and Applications 2.2 (2014), pp. 67-72. 